\title{
Erythropoietin neuroprotection in neonatal cardiac surgery: A phase I/II safety and efficacy trial
}

\author{
Dean B. Andropoulos, MD, MHCM, ${ }^{\mathrm{a}, \mathrm{b}, \mathrm{e}}$ Ken Brady, MD,,${ }^{\mathrm{a}, \mathrm{b}, \mathrm{e}}$ Ronald B. Easley, MD,,${ }^{\mathrm{a}, \mathrm{b}, \mathrm{e}}$ \\ Heather A. Dickerson, MD, ${ }^{\mathrm{b}, \mathrm{g}}$ Robert G. Voigt, MD, ${ }^{\mathrm{b}, \mathrm{k}}$ Lara S. Shekerdemian, MB, BS, ${ }^{\mathrm{b}, \mathrm{h}}$ \\ Marcie R. Meador, BSN, MS, RN, ${ }^{\mathrm{a}, \mathrm{b}, \mathrm{e}}$ Carol A. Eisenman, BSN, RN, ${ }^{\mathrm{b}, \mathrm{e}}$ Jill V. Hunter, MB, BS, ${ }^{\mathrm{d}, \mathrm{i}}$ \\ Marie Turcich, BA, MA, ${ }^{\mathrm{b}, \mathrm{k}}$ Carlos Rivera, MD, ${ }^{\mathrm{b}, \mathrm{j}}$ Emmett D. McKenzie, MD, ${ }^{\mathrm{c}, \mathrm{f}}$ \\ Jeffrey S. Heinle, MD, ${ }^{\mathrm{c}, \mathrm{f}}$ and Charles D. Fraser, Jr, MD ${ }^{\mathrm{b}, \mathrm{c}, \mathrm{f}}$
}

Objectives: Neonates undergoing complex congenital heart surgery have a significant incidence of neurologic problems. Erythropoietin has antiapoptotic, antiexcitatory, and anti-inflammatory properties to prevent neuronal cell death in animal models, and improves neurodevelopmental outcomes in full-term neonates with hypoxic ischemic encephalopathy. We designed a prospective phase I/II trial of erythropoietin neuroprotection in neonatal cardiac surgery to assess safety and indicate efficacy.

Methods: Neonates undergoing surgery for D-transposition of the great vessels, hypoplastic left heart syndrome, or aortic arch reconstruction were randomized to 3 perioperative doses of erythropoietin or placebo. Neurodevelopmental testing using the Bayley Scales of Infant and Toddler Development III was performed at age 12 months.

Results: Fifty-nine patients received the study drug. Safety profile, including magnetic resonance imaging brain injury, clinical events, and death, was not different between groups. Three patients in each group died. Forty-two patients (22 in the erythropoietin group and 20 in the placebo group; 79\% of survivors) returned for 12-month follow-up. In the group receiving erythropoietin, mean Cognitive Scale scores were 101.1 \pm 13.6 , Language Scale scores were $88.5 \pm 12.8$, and Motor Scale scores were $89.9 \pm 12.3$. In the group receiving placebo, Cognitive Scale scores were 106.3 $\pm 10.8(P=.19)$, Language Scores were $92.4 \pm 12.4(P=.33)$, and Motor Scale scores were $92.6 \pm 14.1(P=.51)$.

Conclusions: Safety profile for erythropoietin administration was not different than placebo. Neurodevelopmental outcomes were not different between groups; however, this pilot study was not powered to definitively address this outcome. Lessons learned suggest optimized study design features for a larger prospective trial to definitively address the utility of erythropoietin for neuroprotection in this population. (J Thorac Cardiovasc Surg 2013;146:124-31)

Despite major advances in reducing mortality for neonatal cardiac surgery, acute neurologic morbidity after cardiac surgery (eg, seizures, coma, and movement disorders) occurs in $1 \%$ to $25 \%$ of patients in recent reports. ${ }^{1,2}$ Long-term neurodevelopmental impairment is reported in about half of children undergoing cardiac surgery as

From the Departments of Anesthesiology, ${ }^{\mathrm{a}}$ Pediatrics, ${ }^{\mathrm{b}}$ Surgery, ${ }^{\mathrm{c}}$ and Radiology, ${ }^{\mathrm{d}}$ Baylor College of Medicine, and Divisions of Pediatric Cardiovascular Anesthesiology, ${ }^{\mathrm{e}}$ Congenital Heart Surgery, ${ }^{\mathrm{f}}$ Pediatric Cardiology, ${ }^{\mathrm{g}}$ Pediatric Intensive Care, ${ }^{\mathrm{h}}$ Pediatric Neuroradiology, ${ }^{\mathrm{i}}$ Pediatric Neurology, ${ }^{\mathrm{j}}$ and Developmental Pediatrics, ${ }^{\mathrm{k}}$ Texas Children's Hospital, Houston, Tex.

Supported by National Institutes of Health Eunice Kennedy Shriver National Institute of Child Health and Development grant No. 1R21-HD55501-01, Baylor College of Medicine General Clinical Research Center grant No. 0942 (funded by NIH M01 RR00188), a Charles A. Dana Foundation Brain and Immuno-Imaging Grant, and the Texas Children's Hospital Anesthesiology Research Fund (to D.A.).

Disclosures: Authors have nothing to disclose with regard to commercial support.

Received for publication July 10, 2012; revisions received Aug 26, 2012; accepted for publication Sept 19, 2012; available ahead of print Oct 24, 2012.

Address for reprints: Dean B. Andropoulos, MD, MHCM, Baylor College of Medicine, 6621 Fannin, W 17417, Houston, TX 77030 (E-mail: dra@bcm.tmc.edu).

0022-5223/\$36.00

Copyright (c) 2013 by The American Association for Thoracic Surgery

http://dx.doi.org/10.1016/j.jtcvs.2012.09.046 newborns or young infants. ${ }^{3}$ Data from long-term followup studies in preschool and school-aged children who underwent surgery for congenital heart disease reveal a spectrum of neurodevelopment-related problems that are similar to those observed in low-birth-weight premature infants, including problems with attention, language, memory, and sensorimotor functioning. ${ }^{3}$

Perioperative causes of neurologic injury include cerebral hypoxia/ischemia due to cardiovascular pathophysiology, surgical or cardiopulmonary bypass (CPB) techniques, cerebral emboli, low cardiac output, and intercurrent events such as cardiac arrests., ${ }^{2,5}$ Pre- and postoperative magnetic resonance imaging (MRI) studies document a $36 \%$ to $73 \%$ incidence of new or worsening white matter or other ischemic brain lesions after neonatal cardiac surgery. ${ }^{6,7}$

Erythropoietin (EPO) is a 30.4 kilodalton glycoprotein that has antiapoptotic, anti-inflammatory, and antiexcitatory cell death effects, protecting the brain against a variety of cerebral insults in animal and in vitro models. ${ }^{8,9}$ Human neonatal data show improved longer-term 

Abbreviations and Acronyms
$\mathrm{CPB}=$ cardiopulmonary bypass
$\mathrm{EPO}=$ erythropoietin
FDA $=$ Food and Drug Administration
MRI = magnetic resonance imaging
$\mathrm{rSO}_{2}=$ regional cerebral oxygen saturation

neurodevelopmental outcome with EPO after hypoxic-ischemic encephalopathy. ${ }^{10}$ Because EPO is already used in the neonatal population for prevention and treatment of anemia and has a long record of safety, it is potentially a very useful agent for neuroprotection in neonatal cardiac surgery. The predictable period of known risk of potential neurologic injury in neonatal cardiac surgery makes pre-emptive intervention with EPO to protect the brain an attractive concept. However, the effects of perioperative administration of EPO, in the higher doses needed to provide neuroprotection than those routinely used for anemia, are not known. This phase I/II trial was designed to assess safety and give preliminary indication of efficacy of EPO treatment for neuroprotection in the perioperative period for neonatal cardiac surgery, to help determine if larger trials are warranted.

\section{METHODS}

This was a prospective, randomized, blinded, placebo-controlled trial of EPO (Epogen; Amgen, Inc, Thousand Oaks, Calif) versus normal saline control (FDA IND 100011, NCT00513240, www.clinicaltrials.gov). The Baylor College of Medicine Institutional Review Board approved the protocol, and patients were enrolled after informed signed parental consent.

Inclusion criteria were neonates (aged $<30$ days) scheduled for cardiac surgery with hypothermic CPB for $>60$ minutes. Three anatomic groups were studied: (1) hypoplastic left heart syndrome or variant undergoing Norwood Stage I palliation; (2) D-transposition of the great vessels undergoing arterial switch operation; and (3) interrupted aortic arch with ventricular septal defect or other complete 2-ventricle anatomic repair, including truncus arteriosus, tetralogy of Fallot, or total anomalous pulmonary venous return. Randomization was performed by computer-generated random number assignment to EPO or placebo, and patients were stratified within each of these 3 anatomic groups.

Exclusion criteria were gestational age younger than 35 weeks at birth, weight $<2.0 \mathrm{~kg}$, known recognizable dysmorphic syndrome, surgery not requiring $\mathrm{CPB}$, preoperative cardiac arrest, or inability to enroll the patient $>12$ hours preoperatively. Also excluded were cases where aortic crossclamping was not used, CPB times were anticipated to be $<60$ minutes, and a nadir temperature on bypass $>30^{\circ} \mathrm{C}$ was planned. In addition, patients with contraindications to EPO administration were excluded. Exclusion criteria included hypertension (sustained systolic blood pressure $>100$ $\mathrm{mm} \mathrm{Hg}$ ), polycythemia (hemoglobin $>20 \mathrm{~g} / \mathrm{dL}$ ), thrombocytosis (platelet count $>600,000 / \mathrm{dL}$ ), or evidence of hypercoagulability (international normalized ratio $<1.0$ ), and patient or maternal history of major thrombosis. ${ }^{9}$

Patients underwent a detailed neurologic examination by a pediatric neurologist, unless precluded by neuromuscular blockade or heavy sedation. For a 12- to 24-hour period before the scheduled surgery, patients had a near-infrared spectroscopy sensor (Somanetics 5100B, Inc, Troy, Mich) placed on the right forehead, to measure regional cerebral oxygen saturation $\left(\mathrm{rSO}_{2}\right)$.
Surgical, anesthetic, and CPB techniques were standardized and have been described in detail previously. ${ }^{7}$ Bilateral $\mathrm{rSO}_{2}$ was measured intraoperatively and maintained $>90 \%$ during $\mathrm{CPB}$. If $\mathrm{rSO}_{2}$ was $<50 \%$ before or after bypass, attempts were made to increase oxygen delivery to the brain, or decrease oxygen consumption, using a protocol described previously. ${ }^{7}$

Brain MRI under general endotracheal anesthesia was obtained immediately before surgery. MRI was performed on a 1.5 Tesla Intera scanner (Philips Medical Systems, Best, The Netherlands), including standard T1, T2, diffusion weighted imaging, and susceptibility weighted imaging. ${ }^{7}$ Postoperative MRI was obtained when the patient was clinically stable, at 7 to 10 days postoperatively. All MRI scans were evaluated by pediatric neuroradiologists unaware of diagnosis or surgery. Abnormalities were classified as white matter injury, intraparenchymal infarction, or intraparenchymal or intraventricular hemorrhage. ${ }^{7}$ Sinovenous thrombosis was diagnosed separately, and confirmed by 2-dimensional time of flight magnetic resonance venography with maximum intensity projection reconstructions. MRI injury definitions and grading scale have been described in detail previously; all injuries were classified as mild, moderate, or severe. ${ }^{7}$

The initial protocol EPO doses were 1000 units/kg intravenously over 60 minutes (or placebo equivalent) in 3 doses: (1) 12 to 24 hours preoperatively, (2) immediately after CPB, and (3) 24 hours after dose 2 . This dose was chosen based on animal model data to provide sufficient brain tissue EPO concentrations to afford neuroprotection. ${ }^{11}$ This schema was used in the first 33 patients. The trial was placed on full clinical hold by the US Food and Drug Administration (FDA) because of adverse events in an adult EPO neuroprotection trial for stroke in patients aged 50 to 80 years not related to this neonatal cardiac surgery trial. After evaluation of safety data, the FDA mandated a dosing regimen change in our trial. For the final $26 \mathrm{pa}-$ tients, EPO dose was 500 units/kg intravenously preoperatively, and on postoperative days 1 and 3. Aprotinin was administered to the first 21 patients for antifibrinolysis. Aprotinin marketing was suspended in December 2007, and $\varepsilon$-aminocaproic acid was administered to the final 38 patients in the study.

Clinical data collection preoperatively, intraoperatively, and for the first 72 hours postoperatively included $\mathrm{rSO}_{2}$; hemodynamic and respiratory data; details of CPB technique; and clinical events such as cardiac arrest, extracorporeal membrane oxygenation cannulation, and clinical seizures. The potential adverse effects of EPO include major thrombosis, intracranial hemorrhage, hypertension, thrombocytosis, and polycythemia. ${ }^{9}$ These events were defined as above under Exclusion Criteria, and were assessed for each patient.

Chromosome analysis was performed by chromosomal microarray, or fluorescence in situ hybridization analysis, when a genetic syndrome was suspected. Anesthetic and sedative drug doses in the operating room, and the first 72 postoperative hours in the intensive care unit were recorded. Records were also reviewed for cardiac arrest, extracorporeal membrane oxygenation cannulation, and deaths.

Neurodevelopmental testing was performed with the Bayley Scales of Infant and Toddler Development, Third Edition ${ }^{11}$ (Bayley III) at age 12 months. The Bayley III consists of 3 primary composite standard scores, the Cognitive, Motor, and Language Composite scores, measured by performance of specified tasks, scored against a normative population, and scaled to have a mean score of 100 with standard deviation of 15 . In addition, a parental questionnaire is administered, and Social-Emotional and Adaptive Behavior Composite Scores are derived. These tests were administered by a single developmental psychologist unaware of diagnosis or surgery performed, or if EPO or placebo had been administered. Maternal intelligence was evaluated using the Weschler Abbreviated Scale of Intelligence. $^{12}$

A data and safety monitoring board, consisting of pediatric specialists in neurology, cardiology/cardiac intensive care, congenital heart surgery, hematology, neonatology, and cardiac anesthesiology, reviewed all safety data and adverse events. Blinding of groups was maintained until the final patient had undergone 12-month Bayley III assessment. The data and safety monitoring board had access to unblinded data at all times. 


\section{EPO Pharmacokinetic Data}

Blood sampling for measurement of EPO pharmacokinetics was performed in consenting patients with indwelling arterial or central venous catheters. Sampling $(0.5 \mathrm{~mL})$ was performed immediately before the first dose of EPO/placebo, and at $0,1,2,4,6,12$, and 24 hours after the 1-hour infusion. Immediately following collection, blood was centrifuged at $760 \times \mathrm{g}$ for 15 minutes at room temperature and plasma transferred to plastic vials and frozen at $-80^{\circ} \mathrm{C}$ until analyzed. EPO concentrations were determined using the commercially available Quantikine IVD (R\&D Systems, Inc, Minneapolis, Minn) EPO enzyme-linked immunosorbent assay kit. Because the assay does not discriminate between endogenous and recombinant EPO, the baseline EPO level was subtracted from the post-EPO administration concentrations.

\section{Sample Size Calculation and Statistical Analysis}

This was a pilot phase I/II trial, and the original plan was to enroll $80 \mathrm{pa}-$ tients, to allow for approximately 60 patients to undergo 12-month neurodevelopment assessments, accounting for deaths and withdrawal from the study. This sample size would be expected to detect a difference of 7.5 points between groups on the Cognitive Scale score of the Bayley III, with a standard deviation of 15 , power of $80 \%$, and $\alpha$ level of 0.05 , using 2-sided $t$ test for comparison. Because the time period to enroll patients was significantly curtailed by the full clinical hold imposed by the FDA, the enrollment goal was reduced to 60 , intended to yield 45 patients to evaluate at 12 months. This sample size could only detect a difference of 9 points between groups. Thus this study could only give a preliminary indication of efficacy of EPO treatment on 12-month neurodevelopment-related outcomes.

A descriptive analysis of the pharmacokinetic data was planned due to the study size, and elective participation in the pharmacokinetic analysis component of the study, restricted to infants with indwelling catheters capable of blood sampling in the preoperative period. We anticipated that 10 to 15 patients would meet the inclusion criteria of parental consent, indwelling catheter for blood samples, and ability to enroll the patient $>24$ hours before scheduled surgery.

Analysis was performed on the intention-to-treat basis. The primary analysis was the safety profile for the EPO versus placebo groups, with sinovenous thrombosis, other major thromboses, hypertension, thrombocytosis, and polycythemia as the primary outcomes. Also analyzed were MRI brain injury pre- and postoperatively. Finally, neurodevelopment-related outcomes were analyzed, including Cognitive, Language, and Motor Composite Standard Scores of the Bayley III scales. Data were analyzed using 2-sided $t$ test, Mann-Whitney signed rank test, Fisher exact test, analysis of variance, or $\chi^{2}$ analysis as appropriate. Normally distributed data are reported as mean \pm standard deviation; non-normally distributed data (Shapiro-Wilk test $P<.05$ ) are reported as median (25th-75th percentile). Stata 12 (Stata Corporation LLP, College Station Tex) was used for the analyses.

\section{RESULTS}

The study enrolled patients starting in September 2006, and finished enrollment in February 2011. The enrollment flow diagram is displayed in Figure 1. A total of 104 patients met inclusion criteria. Sixty-two patients $(60 \%$ of those eligible) were enrolled and randomized; 3 patients did not receive intended surgery, and did not have further data collection, leaving 59 patients receiving study drug and perioperative data collection. Patient clinical and perioperative data are presented in Table 1 . All patients survived the immediate postoperative period.

The safety profile was similar between groups. Six patients had postoperative dural sinovenous thrombosis (3 from the EPO group, 3 from the placebo group); and 1 of these, an EPO group patient, had clinical seizures and a new cerebral infarction in the area adjacent to the thrombosis on postoperative MRI. In the other 5 patients, there were no clinical signs and sinovenous thrombosis was only discovered on the postoperative MRI scan. Laboratory workup for hypercoagulability was negative in all cases. One patient with hypoplastic left heart syndrome who received EPO experienced a cardiac arrest and required extracorporeal membrane oxygenation cannulation in the immediate postoperative period. There were no other acute adverse events, including no clinical seizures, coma, major thromboses, hypertension, thrombocytosis, or polycythemia in the immediate postoperative period until hospital discharge. Clinical neurologic examination was performed preoperatively by a pediatric neurologist in 44 of 59 patients, and before discharge in 45 of 59 patients. The only abnormality noted was a slight symmetrical increase in lower extremity deep tendon reflexes on the postoperative examination in 1 patient with transposition of the great arteries receiving placebo who had a moderate new white matter injury, and a new germinal matrix hemorrhage on postoperative MRI.

Six patients died before age 12 months ( 3 from the EPO group, 3 from the placebo group). All patients who died had hypoplastic left heart syndrome; 5 of 6 died in the interstage period before a planned bidirectional cavopulmonary anastomosis, and 1 after. Eleven patients declined 12-month follow-up ( 7 from the EPO group, 4 from the placebo group; $P=.48$ ), leaving 42 patients with 12-month Bayley III scale scores (79\% of survivors).

Brain MRI findings are presented in Table 2. Both preoperative baseline rate of injury, and postoperative outcomes were not different in the EPO patients. There were no differences between groups in the classification of MRI injury severity.

The neurodevelopment-related outcomes are presented in Table 3. The outcomes of Bayley III Cognitive, Language, and Motor Composite scaled scores were not different between groups. We elected to analyze separately the effect of cardiac diagnosis, the change of EPO dose status, and change in aprotinin administration; results are presented in Tables 4 through 6 . The only difference found in these analyses was a higher Bayley III Cognitive Scale score in placebo group patients who did not receive aprotinin. (Table 5) Maternal intelligence quotient for the infants tested at 12 months was $104.5 \pm 14.6$ in the EPO group, and $103.8 \pm 17.2$ in the placebo group $(P=.872)$. The anatomic group of other complete 2-ventricle repairs (ventricular septal defect with aortic arch repair, $\mathrm{n}=5$; truncus repair, $\mathrm{n}=2$; and tetralogy of Fallot and total anomalous pulmonary venous return, $\mathrm{n}=1$ each) had the lowest overall scores. Of note, 6 of 9 patients in this group had chromosome anomalies, all were microdeletions at the chromosome $22 \mathrm{q} 11.2$ region. 


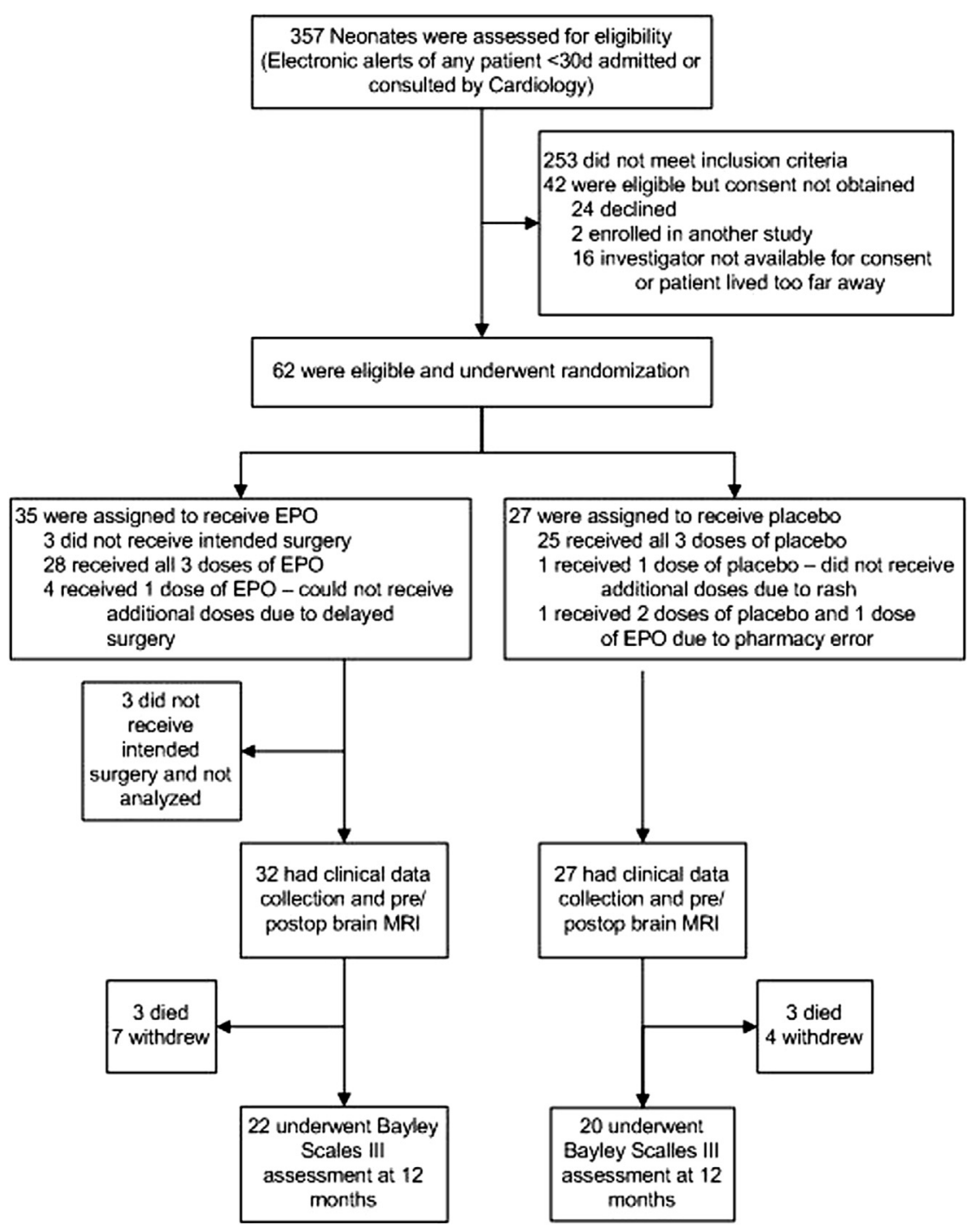

FIGURE 1. Enrollment and data analysis flow diagram.

Three patients had pharmacokinetic data obtained; 1 placebo and 2 patients who received EPO 1000 units/kg. Pharmacokinetic modeling was not performed because of these small patient numbers; however, maximum EPO plasma concentrations were 8415 and $5447 \mathrm{mIU} / \mathrm{mL}$ in the 2 patients receiving EPO.

\section{DISCUSSION}

EPO treatment was not associated with an overall difference in complications, including major intracranial thromboses, hemorrhage, and other MRI findings; clinical adverse outcomes; and death. Twelve-month Bayley III scale scores were not different with EPO treatment; because of the phase I/II pilot study design, this trial was not powered to demonstrate a neurodevelopmental outcome difference. Maximum EPO plasma levels in the 2 patients where they were obtained demonstrated concentrations consistent with neuroprotective levels in animal models for 1 patient, and concentration below these levels in the other. ${ }^{13}$

This EPO neuroprotection study is the first published study in the neonatal and infant cardiac surgery population to apply a pharmacologic neuroprotection strategy, measure cerebral physiology with near infrared spectroscopy, evaluate the structural changes of the brain with MRI, and assess the functional outcomes with longer-term neurodevelopment-related outcomes at age 12 months. The only previously published randomized trial of pharmacologic neuroprotection in this population was a study by Clancy and colleagues ${ }^{14}$ of allopurinol as an oxygen free radical scavenger versus placebo for neonates undergoing deep hypothermic circulatory arrest for Norwood stage I palliation or other complex repairs. There was no effect of allopurinol treatment on the primary neurologic outcome (ie, seizures, coma, or death). When cardiac outcomes were included, the hypoplastic left heart syndrome 
TABLE 1. Patient and operative characteristics

\begin{tabular}{|c|c|c|c|}
\hline Parameter & EPO group $(n=32)$ & Placebo group $(n=27)$ & $P$ value \\
\hline HLHS (n, \%) & $16(50)$ & $10(37)$ & .420 \\
\hline D-TGA (n, \%) & $9(28)$ & $12(44)$ & - \\
\hline $\mathrm{AA}+\mathrm{VSD} /$ other $2 \mathrm{~V}(\mathrm{n}, \%)$ & $7(22)$ & $5(19)$ & - \\
\hline CPB time $(\min )^{*}$ & $191(163-236)$ & $194(169-270)$ & .382 \\
\hline Aortic crossclamp time (min)* & $98(84-129)$ & $104(89-148)$ & .334 \\
\hline DHCA time $(\min )^{*}$ & $9(8-14)(\mathrm{n}=19)$ & $11(8-14)(n=10)$ & .562 \\
\hline RCP time (min) & $67.0 \pm 30.7(\mathrm{n}=23)$ & $67.9 \pm 31.2(\mathrm{n}=14)$ & .930 \\
\hline Lowest CPB temperature $\left({ }^{\circ} \mathrm{C}\right)^{*}$ & $17.9(17.4-22.8)$ & $18.1(17.6-24.9)$ & .266 \\
\hline OR fentanyl dose $(\mu \mathrm{g} / \mathrm{kg})^{*}$ & $182(154-291)$ & $198(158-295)$ & .755 \\
\hline OR midazolam dose $(\mathrm{mg} / \mathrm{kg})$ & $0.96 \pm 0.46$ & $1.21 \pm 0.49$ & $.044 \dagger$ \\
\hline Aprotinin use (n, \%) & $11(34)$ & $10(37)$ & .952 \\
\hline Isoflurane MAC-hours & $1.78(0.96-2.29)$ & $1.58(1.14-2.68)$ & .879 \\
\hline Mean $\mathrm{rSO}_{2}(\%)$ & $69.6 \pm 7.1$ & $71.2 \pm 7.4$ & .394 \\
\hline
\end{tabular}

Data are mean \pm standard deviation if normally distributed. $E P O$, Erythropoietin; $H L H S$, hypoplastic left heart syndrome; $D$-TGA, dextrotransposition of the great arteries; $A A+V S D$, hypoplastic aortic arch/ventricular septal defect; $2 V, 2$ ventricle; $C P B$, cardiopulmonary bypass; $O R$, operating room; $D H C A$, deep hypothermic circulatory arrest; $R C P$, regional cerebral perfusion; $M A C$, minimum alveolar concentration; $r \mathrm{SO}_{2}$, regional cerebral oxygen saturation. *Data not normally distributed: median (25th-75th percentiles). $\dagger P<.05$.

group did have an improvement with allopurinol treatment. No imaging or longer-term outcomes were assessed.

EPO treatment shows promise to improve neurodevelopment-related outcomes in other clinical settings of neonatal brain injury. A prospective randomized controlled trial in 153 full-term neonates diagnosed with hypoxic ischemic encephalopathy demonstrated an improvement in neurodevelopment-related outcomes at age 18 months after EPO treatment every other day for 2 weeks. ${ }^{10}$ Death or moderate/severe disability occurred in $44 \%$ infants in the control group and $25 \%$ infants in the EPO group $(P=.017)$. EPO improved outcomes only for infants with moderate hypoxic ischemic encephalopathy $(P=.001)$ and not those with severe injury $(P=.227)$ A second small case control trial $(n=45)$ in birth asphyxiated full term neonates found that EPO, 2500 units $/ \mathrm{kg}$ subcutaneously for 5 days, resulted in fewer neurologic and developmental abnormalities at age 6 months than control patients $(27 \%$ vs $71 \% ; P=.03){ }^{15}$ In an EPO neuroprotection study in extremely low-birth-weight neonates $(<1000 \mathrm{~g}), 17$ survivors received either 500,1000, or 2500 units $/ \mathrm{kg}$ intravenous EPO daily for the first 3 days of life. Compared with the 18 control subjects receiving no EPO, both cognitive $(P=.044)$ and motor $(P=.026)$ scale scores were higher with EPO, from 8 to 36 months corrected gestational age. ${ }^{16}$

There are recent data demonstrating apoptosis in response to common anesthetic agents that bind $\gamma$-aminobutyric acid and N-methyl-D-aspartate receptors to produce their effects, including halogenated anesthetic gases, benzodiazepines, and ketamine. These drugs are frequently used in large doses

TABLE 2. Brain magnetic resonance imaging (MRI) findings

\begin{tabular}{|c|c|c|c|c|}
\hline Time period & Parameter & EPO group $(\mathbf{n}=32)$ & Placebo group $(n=27)$ & $P$ value \\
\hline \multirow[t]{8}{*}{ Preoperative MRI injury } & Total with WMI, infarction, or hemorrhage (n, \%) & $13(41)$ & $9(33)$ & .759 \\
\hline & WMI & $9(28)$ & $8(30)$ & .872 \\
\hline & WMI severity: mild/moderate/severe (n) & $6 / 2 / 1$ & $6 / 2 / 0$ & - \\
\hline & Infarction $(\mathrm{n}, \%)$ & $6(19)$ & $2(7)$ & .269 \\
\hline & Infarction severity: mild/moderate/severe (n) & $4 / 1 / 1$ & $2 / 0 / 0$ & - \\
\hline & Hemorrhage $(\mathrm{n}, \%)$ & $3(9)$ & $3(11)$ & .652 \\
\hline & Hemorrhage severity: mild/moderate/severe (n) & $3 / 0 / 0$ & $3 / 0 / 0$ & - \\
\hline & DSVT & 0 & 0 & - \\
\hline \multirow[t]{9}{*}{ New postoperative MRI injury } & Total with new WMI, infarction, or hemorrhage & $13(41)$ & $13(48)$ & .751 \\
\hline & WMI (n, \%) & $8(25)$ & $9(33)$ & .678 \\
\hline & WMI severity: mild/moderate/severe (n) & $6 / 2 / 0$ & $6 / 3 / 0$ & - \\
\hline & Infarction $(\mathrm{n}, \%)$ & $3(9)$ & $5(19)$ & .450 \\
\hline & Infarction severity: mild/moderate/severe (n) & $3 / 0 / 0$ & $5 / 0 / 0$ & - \\
\hline & Hemorrhage (n, \%) & $7(22)$ & $3(11)$ & .319 \\
\hline & Hemorrhage severity: mild/moderate/severe (n) & $6 / 0 / 1$ & $3 / 0 / 0$ & - \\
\hline & $\operatorname{DSVT}(\mathrm{n}, \%)$ & $3(9)$ & $3(11)$ & .997 \\
\hline & DSVT severity: mild/moderate/severe (n) & $2 / 1 / 0$ & $2 / 1 / 0$ & - \\
\hline
\end{tabular}

Some patients had more than 1 type of injury, resulting in totals of WMI, infarction, and hemorrhage in each group being greater than the total number of brain-injured patients. $E P O$, Erythropoietin; WMI, white matter injury; DSVT, dural sinovenous thrombosis. 
TABLE 3. Neurodevelopmental outcomes: 12 month Bayley Scales of Infant and Toddler Development III (Bayley III) Composite Scaled Scores

\begin{tabular}{|c|c|c|c|c|}
\hline Variable & Parameter & EPO group $(n=22)$ & Placebo group $(\mathbf{n}=20)$ & $P$ value \\
\hline \multirow[t]{3}{*}{ Cardiac diagnosis } & HLHS (n, \%) & $11(50)$ & $6(30)$ & .298 \\
\hline & D-TGA $(n, \%)$ & $6(27)$ & $10(50)$ & - \\
\hline & $\mathrm{AA}+\mathrm{VSD} /$ other $2 \mathrm{~V}(\mathrm{n}, \%)$ & $5(23)$ & $4(20)$ & - \\
\hline \multirow{3}{*}{$\begin{array}{l}\text { Directly measured Bayley III } \\
\text { composite scores }\end{array}$} & Cognitive & $101.1 \pm 13.6$ & $106.3 \pm 10.8$ & .187 \\
\hline & Language & $88.5 \pm 12.8$ & $92.4 \pm 12.4$ & .329 \\
\hline & Motor & $89.9 \pm 12.3$ & $92.6 \pm 14.1$ & .506 \\
\hline \multirow{5}{*}{$\begin{array}{l}\text { Adaptive behavior: Bayley III } \\
\text { questionnaire scores }\end{array}$} & Social-emotional* & $95.0(92.5-105.0)$ & $100.0(96.3-108.8)$ & .249 \\
\hline & Behavioral & $93.2 \pm 10.7$ & $97.3 \pm 15.7$ & .342 \\
\hline & Conceptual & $98.7 \pm 13.6$ & $99.2 \pm 13.1$ & .906 \\
\hline & Social & $97.2 \pm 11.4$ & $100.7 \pm 15.6$ & .423 \\
\hline & Practical & $89.5 \pm 9.1$ & $92.8 \pm 12.6$ & .352 \\
\hline
\end{tabular}

Data are mean \pm standard deviation if normally distributed. EPO, Erythropoietin; HLHS, hypoplastic left heart syndrome; $D$-TGA, dextrotransposition of the great arteries; $A A+V S D$, hypoplastic aortic arch/ventricular septal defect; $2 V, 2$ ventricle. *Data not normally distributed: median (25th-75th percentiles).

in neonatal cardiac surgery, and increased exposure to these agents is associated with worse neurodevelopment-related outcomes in neonatal cardiac surgery, even after adjustment for important covariates. ${ }^{17}$ There is now animal model data demonstrating that EPO protects against anesthetic-induced apoptosis and that anesthetics themselves suppress endogenous EPO response to hypoxia. ${ }^{18}$ These potential benefits of EPO treatment, as well as the demonstration of adequate safety profiles of EPO in our trial, suggest that EPO in adequate doses should be studied in a larger cohort of patients undergoing neonatal cardiac surgery, with uniform dosing and treatment protocols, to determine its value as a neuroprotectant in this setting.

Limitations of this study that affect the conclusions reached with regard to the original intent of this phase I/II trial can be summarized as follows. First is the change in EPO dosing to levels that may not be neuroprotective. Second is the reduction in the planned number of patients due to the limited study period available. Third, timing of EPO dosing with regard to the potential insult: a broader dosing window could be more desirable, given the evidence for significant incidence of preoperative MRI brain injury, and the possibility that injury occurred later than postoperative day 3 . Fourth, the lack of adequate numbers of patients for pharmacokinetic data, and lack of data with regard to EPO pharmacokinetics with CPB. Finally, 6 of 42 patients had chromosome 22q11.2 partial deletion, a condition with known but variable associations with problems of neurodevelopment. ${ }^{19}$

The limitations noted above, and the small size of this EPO study preclude making any conclusions about EPO neuroprotection in neonatal cardiac surgery populations. However, as a phase I/II trial it is useful in that it demonstrates no safety concerns of higher dose EPO administration, and gives important insight into optimized study design features for a future EPO neuroprotection trial. Besides studying a population large enough to determine a meaningful difference in the primary neurodevelopment outcome measure, future trials should include (1) more optimal EPO dosing, with the higher doses more likely to provide benefit; (2) more complete pharmacokinetic data, necessary because of the unique features in this population (ie, bypass); (3) more effective exclusion criteria and/or screening to account for enrollment of subjects with chromosome 22q11.2 microdeletions and other chromosomal syndromes, at least assuring equivalent numbers in each treatment group because these patients clearly affect the outcomes $^{19}$; (4) a plan for stratification based on rate of preoperative MRI injury; (5) refinement of the EPO dosing regimen given the issue of preoperative injury, with

TABLE 4. Bayley Scales of Infant and Toddler Development III (Bayley III) scores by anatomic/surgical group

\begin{tabular}{|c|c|c|c|}
\hline Parameter & EPO group & Placebo group & $P$ value \\
\hline 12-Month Bayley III Cognitive HLHS* $(\mathrm{n}=11 \mathrm{EPO}, 6$ placebo $)$ & $100(87.5-113.8)$ & $100(100-100)$ & .990 \\
\hline 12-Month Bayley III Language HLHS & $90.3 \pm 15.1$ & $90.0 \pm 9.7$ & .969 \\
\hline 12-Month Bayley III Motor HLHS* & $91.0(91.0-97.0)$ & $92.5(79.0-110.0)$ & 919 \\
\hline 12-Month Bayley III Cognitive D-TGA ( $\mathrm{n}=7 \mathrm{EPO}, 9$ placebo $)$ & $103.6 \pm 14.1$ & $110.0 \pm 11.7$ & .335 \\
\hline 12-Month Bayley III Language D-TGA* & $91(84.5-97.8)$ & $94(92.8-98.5)$ & .284 \\
\hline 12-Month Bayley III Motor D-TGA & $92.4 \pm 12.0$ & $97.3 \pm 12.3$ & .394 \\
\hline 12-Month Bayley III Cognitive AA+VSD/other 2V $(\mathrm{n}=4 \mathrm{EPO}, 5$ placebo $)$ & $97.5 \pm 6.4$ & $107.0 \pm 13.0$ & .228 \\
\hline 12-Month Bayley III Language AA+VSD/other 2V* & $78.5(69.5-87.5)$ & $91.0(76.3-91.8)$ & .190 \\
\hline 12 month Bayley III Motor AA+VSD/other 2V & $85.0 \pm 12.7$ & $86.8 \pm 11.7$ & .832 \\
\hline
\end{tabular}

Data are mean \pm standard deviation if normally distributed. EPO, Erythropoietin; HLHS, hypoplastic left heart syndrome; $D$-TGA, dextrotransposition of the great arteries; $A A+V S D$, hypoplastic aortic arch/ventricular septal defect; $2 V, 2$ ventricle. *Data not normally distributed: median (25th-75th percentiles). 
TABLE 5. Bayley Scales of Infant and Toddler Development III (Bayley III) scale scores by erythropoietin (EPO) dose: 1000 units/kg every day (1000 QD ) for 3 doses versus 500 units/kg every other day (500 QOD) for 3 doses versus placebo

\begin{tabular}{|c|c|c|c|c|}
\hline Parameter & EPO 1000 QD $(n=11)$ & EPO 500 QOD $(n=11)$ & Placebo $(\mathbf{n}=\mathbf{2 0})$ & $P$ value \\
\hline 12-Month Bayley III Cognitive & $101.4 \pm 16.9$ & $100.9 \pm 10.2$ & $106.3 \pm 10.7$ & 422 \\
\hline 12-Month Bayley III Language & $85.0 \pm 16.3$ & $92.0 \pm 7.3$ & $92.4 \pm 12.4$ & .268 \\
\hline 12-Month Bayley III Motor & $89.3 \pm 15.7$ & $90.5 \pm 8.6$ & $92.6 \pm 14.1$ & .787 \\
\hline
\end{tabular}

Comparison by 1-way analysis of variance.

consideration to starting EPO dosing shortly after birth/diagnosis/randomization, and a longer period of treatment (ie, up to 2 weeks); (6) an optimal primary outcome variable: timing of neurodevelopmental testing at 12 months versus later for greater predictive ability, ${ }^{20}$ issues with Bayley III calibration (newer version of the test yields higher Cognitive Scale score than the Mental Development Index of the Bayley II), ${ }^{21}$ and utility of MRI (recent data demonstrates an association with perioperative MRI brain injury and lower neurodevelopment scores) $)^{22}$; and (7) stratification and randomization according to anatomic diagnoses with known worse neurodevelopment-related outcomes (ie, hypoplastic left heart syndrome vs other diagnoses). ${ }^{23}$

Sample size considerations for an optimized EPO study include choosing an appropriate primary outcome variable; that is, the Cognitive Composite Score of the Bayley III at age 18 to 24 months, powered to detect a difference of 5 points higher with EPO treatment. Assuming a power of $85 \%$ and $\alpha$ level of $0.05,326$ evaluable subjects would be required, using 2-sided $t$ test for comparison. This in turn would likely mean that 435 patients would be required to enroll in the study, accounting for a $25 \%$ death or study dropout rate. This number would increase with stratification by anatomic subgroup, or for interim analyses for safety purposes. Such a study very likely requires multicenter participation to enroll patients over a brief enough window of time to ensure that outcomes do not change substantially because of other unrelated improvements in therapy.

TABLE 6. Bayley Scales of Infant and Toddler Development III (Bayley III) scale scores by aprotinin administration status

\begin{tabular}{|c|c|c|c|}
\hline Parameter & EPO group & Placebo group & $P$ value \\
\hline $\begin{array}{l}\text { 12-Month Bayley III Cognitive } \\
\text { with aprotinin } \\
\text { ( } \mathrm{n}=8 \mathrm{EPO}, 8 \text { placebo) }\end{array}$ & $106.9 \pm 15.3$ & $103.1 \pm 12.8$ & .604 \\
\hline $\begin{array}{l}\text { 12-Month Bayley III Language } \\
\text { with aprotinin }\end{array}$ & $88.1 \pm 14.8$ & $87.4 \pm 8.3$ & .902 \\
\hline $\begin{array}{l}\text { 12-Month Bayley III Motor } \\
\text { with aprotinin }\end{array}$ & $94.3 \pm 12.3$ & $87.3 \pm 15.3$ & .335 \\
\hline $\begin{array}{l}\text { 12-Month Bayley III } \\
\text { Cognitive no aprotinin } \\
\text { (n = } 14 \text { EPO, } 12 \text { placebo) }\end{array}$ & $97.9 \pm 11.9$ & $108.3 \pm 9.1$ & $.020 *$ \\
\hline $\begin{array}{l}\text { 12-Month Bayley III Language } \\
\text { no aprotinin }\end{array}$ & $88.7 \pm 12.1$ & $95.7 \pm 13.8$ & .184 \\
\hline $\begin{array}{l}\text { 12-Month Bayley III Motor } \\
\text { no aprotinin }\end{array}$ & $87.4 \pm 12.1$ & $96.2 \pm 12.4$ & .080 \\
\hline
\end{tabular}

\section{CONCLUSIONS}

In this phase I/II trial, EPO treatment as a neuroprotectant for complex neonatal cardiac surgery was not associated with a different safety profile than placebo, including major intracranial thromboses, hemorrhage, other MRI injuries, and death. EPO treatment was not associated with an overall difference in 12-month Bayley III scale scores in this small group after analysis by the intent-to-treat basis; this study was not powered to demonstrate an outcome difference. Because of the limitations of changing EPO dose and aprotinin strategy during the study, a larger controlled trial would be required to definitively address the status of EPO neuroprotection for neonatal cardiac surgery. In addition, the significant incidence of well-established preoperative brain injury could potentially be addressed with earlier EPO treatment; that is, immediately after birth if the patient had a prenatal diagnosis. Because we observed no differences in adverse effects with EPO 1000 units/kg versus 500 units/ $\mathrm{kg}$, and doses of at least 1000 units/kg are likely to be needed for neuroprotection, we advocate a higher dose EPO strategy for future trials in neonatal cardiac surgery. An optimized study design, likely in a multicenter setting, will be required to define the utility of EPO neuroprotection in neonatal cardiac surgery populations. We advocate the pursuit of such a study because of the many desirable properties of EPO for neuroprotection and its demonstrated efficacy in other setting of neonatal cerebral injury.

The authors acknowledge the Data and Safety Monitoring Board: Donna Ferriero (Chair), Stephen Roth (Secretary), Robin Ohls, Emad Mossad, James DiNardo, Patti Massicotte, Tom Karl, and J. William Gaynor.

\section{References}

1. Menache CC, du Plessis AJ, Wessel DL, Jonas RA, Newburger JW. Current incidence of acute neurologic complications after open-heart operations in children. Ann Thorac Surg. 2002;73:1752-8.

2. Austin EH 3rd, Edmonds HL Jr, Auden SM, Seremet V, Niznik G, Sehic A, et al. Benefit of neurophysiologic monitoring for pediatric cardiac surgery. $J$ Thorac Cardiovasc Surg. 1997;114:707-15.

3. Snookes SH, Gunn JK, Eldridge BJ, Donath SM, Hunt RW, Galea MP, et al. A systematic review of motor and cognitive outcomes after early surgery for congenital heart disease. Pediatrics. 2010;125:e818-27.

4. Jonas RA, Wypij D, Roth SJ, Bellinger DC, Visconti KJ, du Plessis AJ, et al. The influence of hemodilution on outcome after hypothermic cardiopulmonary bypass: results of a randomized trial in infants. J Thorac Cardiovasc Surg. 2003;126:1765-74.

5. Wypij D, Newburger JW, Rappaport LA, duPlessis AJ, Jonas RA, Wernovsky G, et al. The effect of duration of deep hypothermic circulatory arrest in infant heart surgery on late neurodevelopment: the Boston Circulatory Arrest Trial. J Thorac Cardiovasc Surg. 2003;126:1397-403. 
6. McQuillen PS, Barkovich AJ, Hamrick SE, Perez M, Ward P, Glidden DV, et al. Temporal and anatomic risk profile of brain injury with neonatal repair of congenital heart defects. Stroke. 2007;38:736-41.

7. Andropoulos DB, Hunter JV, Nelson DP, Stayer SA, Stark AR, McKenzie ED, et al. Brain immaturity is associated with brain injury before and after neonatal cardiac surgery with high-flow bypass and cerebral oxygenation monitoring. J Thorac Cardiovasc Surg. 2010;139:543-56.

8. McPherson RJ, Juul SE. Erythropoietin for infants with hypoxic-ischemic encephalopathy. Curr Opin Pediatr. 2010;22:139-45.

9. Xiong T, Qu Y, Mu D, Ferriero D. Erythropoietin for neonatal brain injury: opportunity and challenge. Int J Dev Neurosci. 2011;29:583-91.

10. Zhu C, Kang W, Xu F, Cheng X, Zhang Z, Jia L, et al. Erythropoietin improved neurologic outcomes in newborns with hypoxic-ischemic encephalopathy. Pediatrics. 2009;124:e218-26.

11. Bayley N. Bayley Scales of Infant and Toddler Development-third edition. San Antonio, TX: Person Assessment; 2006.

12. Weschler D. Weschler Abbreviated Scale of Intelligence. Antonio, TX: Person Assessment; 1999.

13. Statler PA, McPherson RJ, Bauer LA, Kellert BA, Juul SE. Pharmacokinetics of high-dose recombinant erythropoietin in plasma and brain of neonatal rats. Pediatr Res. 2007;61:671-5.

14. Clancy RR, McGaurn SA, Goin JE, Hirtz DG, Norwood WI, Gaynor JW, et al. Allopurinol neurocardiac protection trial in infants undergoing heart surgery using deep hypothermic circulatory arrest. Pediatrics. 2001;108:61-70.

15. Elmahdy H, El-Mashad AR, El-Bahrawy H, El-Gohary T, El-Barbary A, Aly H. Human recombinant erythropoietin in asphyxia neonatorum: pilot trial. Pediatrics. 2010;125:e1135-42.
16. McAdams RM, McPherson RJ, Mayock DE, Juul SE. Outcomes of extremely low birth weight infants given early high-dose erythropoietin. $J$ Perinatol. June 21, 2012 [Epub ahead of print].

17. Andropoulos DB, Easley RB, Brady K, Stayer S, Meador M, Eisenman C, et al. Cumulative volatile anesthetic exposure and neurodevelopmental outcomes at age 12 months after neonatal cardiac surgery. Anesthesiology. 2012;117:A798

18. Tanaka T, Kai S, Koyama T, Daijo H, Adachi T, Fukuda K, et al. General anesthetics inhibit erythropoietin induction under hypoxic conditions in the mouse brain. PLoS One. 2011;6(12):e29378.

19. Atallah J, Joffe AR, Robertson CM, Leonard N, Blakley PM, Nettel-Aguirre A et al. Two-year general and neurodevelopmental outcome after neonatal complex cardiac surgery in patients with deletion 22q11.2: a comparative study. $J$ Thorac Cardiovasc Surg. 2007;134:772-9.

20. Harris SR, Megens AM, Backman CL, Hayes VE. Stability of the Bayley II Scales of Infant Development in a sample of low-risk and high-risk infants. Dev Med Child Neurol. 2005;47:820-3.

21. Lowe JR, Erickson SJ, Schrader R, Duncan AF. Comparison of the Bayley II Mental Developmental Index and the Bayley III Cognitive Scale: are we measuring the same thing? Acta Paediatr. 2012;101:e55-8.

22. Andropoulos DB, Easley RB, Brady K, McKenzie ED, Heinle JS, Dickerson HA et al. Changing expectations for neurological outcomes after the neonatal arteria switch operation. Ann Thorac Surg. 2012;94:1250-6.

23. Gaynor JW, Gerdes M, Nord AS, Bernbaum J, Zackai E, Wernovsky G, et al. Is cardiac diagnosis a predictor of neurodevelopmental outcome after cardiac surgery in infancy? J Thorac Cardiovasc Surg. 2010;140: 1230-7 